\title{
Variability of Radio AGN in the CHILES Field
}

\author{
Charee Peters ${ }^{1,2}$, Laura Chomiuk $^{3}$, Evangelia Tremou ${ }^{3}$, and Eric Wilcots ${ }^{1}$ \\ ${ }^{1}$ University of Wisconsin-Madison, $475 \mathrm{~N}$. Charter \\ Street, Madison, WI 53706-1507, USA \\ ${ }^{2}$ LSSTC Data Science Fellow \\ ${ }^{3}$ Michigan State University, 567 Wilson Road, East \\ Lansing, MI 48824, USA
}

\begin{abstract}
It is clear from previous and ongoing surveys that there are many sources that change in brightness over time. There is a wide range of extragalactic phenomena that create events with time-varying brightness. Variable sources have regular changes in brightness, such as the steady accretion of matter onto a super massive black hole at the center of a galaxy, known as active galactic nuclei (AGN). Despite strongly emitting in radio wavelengths, the radio sky is nearly completely unexplored in terms of these variable events. Most radio surveys on AGN variability have not been deep enough, include a small sample, and only have a few observations to understand how AGN are changing on timescales between weeks and months. Improving our understanding of the fundamental nature these sources requires a deep radio continuum survey with extension data to observe changes happening on timescales longer than days and less than years. The combination of the COSMOS HI Large Extragalactic Survey (CHILES) and 14 hours of extension observations, I will begin to answer: what is the variability of radio AGN and can it be used to predict and classify future events?
\end{abstract}

\section{Introduction}

The era of time domain studies is nearly upon us. With telescopes like the Large Synoptic Survey Telescope (LSST) and the Zwicky Transient Facility (ZTF), the dynamic, time-domain sky will rapidly open up, revealing some of the most energetic and enigmatic phenomena in the universe. It is clear from previous and ongoing surveys that there are many sources that change in luminosity over time. The time scales over which sources change differ dramatically, from milliseconds to tens of years or longer depending on the type of phenomena occurring.

There is a wide range of extragalactic phenomena that create events with time-varying brightnesses, and these are broken into two types: transient and variable. Transient events mark a discrete episode (or event), wherein the brightness of a source drastically changes and fades out over time. Examples of transients include gamma-ray bursts (GRBs), the deaths of stars as explosive supernovae (SNe), and tidal disruption events (TDEs), events when a star is ripped apart by a black hole (e.g., Rees (1988)). Variable sources refer to objects that have regular or aperiodic changes in brightness, such as active galactic nuclei (AGN), the accretion of matter onto a super massive black hole at the center of a galaxy.

Differentiating between the types of sources responsible for these changes in brightness can be a difficult task, especially when the time scales and amplitudes of brightness on which the changes occur are similar. We need to understand the full range of variability of these sources, so that we can more accurately classify these events and understand the underlying physics creating their variable brightness. Active galactic nuclei (AGN) are numerous and one of the most prominent 
variable events in the radio sky. Although AGN have been well studied in other wavelengths, the variability of AGN in radio wavelengths is relatively unexplored, as previous surveys suffer from low sample sizes and sparse sampling. The question that I propose to answer is: What is the full range of AGN radio variability on a variety of timescales? By answering this, I can work towards the larger question of how the variability can be used to most effectively differentiate between supernovae (SNe), tidal disruption events (TDEs), and AGN?

1.1 Current Status of Radio Time Domain The radio sky is nearly completely unexplored in the time domain. Most blind radio transient surveys have turned-up non-detections because they have not been deep enough or over a wide range of time scales (Frail et al. 2012; Mooley et al. 2016; Hancock et al. 2016). There are many events that are expected to emit radio variability or transient behavior, including AGN, SNe, TDEs, extreme scattering events (Fiedler et al. 1994), and potentially even gravitational wave creating events (binary neutron star mergers and black hole-neutron star mergers) (Hotokezaka et al. 2016). Improving our understanding of the nature of radio transients and variable sources requires a deep radio continuum survey with a large range of observation cadences.

Sparse sampling has made it difficult to quantify what the expected range of variability should be or what the density of variable and transient events are in the radio sky. There is a tradeoff between having a large number of observing epochs over shorter time scales and the sensitivity of the individual epochs. In order to be sensitive to lower luminosity variables, observations are only carried out within a few epochs (e.g., Hancock et al. (2016); Falcke et al. (2001)). Often, this also means that the sample sizes are small and comprised of bright, hand-selected sources (e.g., Barvainis et al. (2005); Mundell et al. (2009)).

The few surveys that have been conducted have one common conclusion: more observations are needed to better understand the radio transient and variable sky. Fortunately, a new $1.4 \mathrm{GHz}$ survey is being conducted at the Karl G. Jansky Very Large Array (VLA), the COSMOS (Cosmic Evolution Survey) HI Large Extragalactic Survey (CHILES), and offers a unique opportunity to study some of the many questions surrounding radio transient and variable events.

1.2 CHILES VERDES and Extension Data CHILES is pointing along a portion of the COSMOS field (Fernández et al. 2013). The is an extraordinarily sensitive radio continuum telescope and the CHILES deep field will yield the deepest radio continuum survey of the COSMOS field to date. There will be a total of 334 epochs of 3-hour integrations, with each epoch being able to be broken up into timescales as short as 10 seconds. When the CHILES data is compared with previous observations from the VLA - COSMOS (observed in 2004), it can probe timescales as long as 14 years. CHILES observes 431 sources at a single time, many being AGN. CHILES provides us with an unprecedented data set with which to study the variability of the radio sky. Although CHILES is primarily invested in spectral line observations of HI at intermediate redshifts (out to $\mathrm{z}=0.45$ ), the new upgrades for the VLA also allow for simultaneous wide-band continuum observations spanning $0.9-1.8 \mathrm{GHz}$; CHILES has two spectral windows that are dedicated for continuum science. With a goal of 1000 hours of observations, and over 400 hours already achieved, reaching a root mean square noise of $\sim 0.5 \mu \mathrm{Jy}$ (Hales et al. 2017, in prep), CHILES provides deep continuum observations spread over year-long timescales, which is ideal for studying the variability of the radio sky, and has led to our project: CHILES VERDES 
(Variable and Explosive Radio Dynamic Evolution Survey).

Although CHILES VERDES has set the stage for unparalleled observations of long-term radio variability and it has the sensitivity to observe events previously undetectable, there are significant, yearlong gaps between 4-month observing blocks (the time between VLA B configurations). Previous radio variability studies have focused on a limited sample of very bright AGN, and very little is known about variability of the lower-luminosity radio sources that dominate modern surveys. It can be difficult to characterize and understand the processes leading to variability on week to month-long timescales when only observing for four months at a time.

As a primary investigator, I was awarded time to continue observing the CHILES field in the Aconfiguration following the CHILES observations to extend the light curves of AGN for continuous weekly coverage for nearly eight months (May 2016 through late January 2017). I observed the CHILES field in one-hour scheduling blocks, spaced roughly once a week throughout the A array (four months), for a total of 14 hours. These observations have been completed and I am currently reducing the data to combine with the CHILES observations. Within a single observing block, all sources within the CHILES field of view are monitored. These weekly observations will ensure coverage needed where previous studies have not probed.

From the combination of CHILES and the extension data, I will perform statistical analysis of the variable light curves to determine any common light curve shapes and the typical behavior of sources at this wavelength. While conducting the statistical analysis, I will also search for any unique or exciting events detected, which may be interesting enough to warrant more detailed analysis. Detecting and understanding the behavior of radio sources will become a more prominent issue as we dive farther into the era of wide field synoptic surveys. This project will offer the unique opportunity to get a head start on understanding common events and trends in AGN variability observed in such surveys.

1.3 Outline of Project There are currently many questions that have yet to be explored with respect to the radio time domain. I am using CHILES observations to conduct a statistical analysis of a blind radio survey and will later focus on individual events to better understand general trends in radio transient and variable events. I have also conducted my own VLA observations to extend CHILES light curves to better understand the full range of low-luminosity AGN; these observations may be key in classifying AGN from TDEs and other transient events.

\section{Acknowledgments}

This material is based upon work supported by NASA under Award No. RFP17_6.0 \#NNX15AJ12H issued through Wisconsin Space Grant Consortium. Any opinions, findings, and conclusions or recommendations expressed in this material are those of the author(s) and do not necessarily reflect the views of the National Aeronautics and Space Administration. It was also supported by the University of Wisconsin -- Madison's Advanced Opportunities Fellowship (AOF), National Science Foundation under the Grant AST-1413099, and the Research Corporation for Science Advancement grant. Charee Peters thanks the LSSTC Data Science Fellowship Program, her time as a Fellow has benefited this work. 


\section{References}

Barvainis R., Lehár J., Birkinshaw M., Falcke H., Blundell K. M., 2005, ApJ, 618, 108

Falcke H., Lehár J., Barvainis R., Nagar N. M., Wilson A. S., 2001, in Peterson B. M., Pogge R. W., Polidan R. S., eds, Astronomical Society of the Pacific Conference Series Vol. 224, Probing the Physics of Active Galactic Nuclei. p. 265 (arXiv:astro-ph/0009457)

Fernández, X., van Gorkom, J. H., Hess, K. M., et al. 2013, ApJ, 770, L29

Fiedler R., Dennison B., Johnston K. J., Waltman E. B., Simon R. S., 1994, ApJ, 430, 581

Frail D. A., Kulkarni S. R., Ofek E. O., Bower G. C., Nakar E., 2012, ApJ, 747, 70

Hancock P. J., Drury J. A., Bell M. E., Murphy T., Gaensler B. M., 2016, MNRAS, 461, 3314

Hotokezaka K., Nissanke S., Hallinan G., Lazio T. J. W., Nakar E., Piran T., 2016, preprint, (arXiv:1605.09395)

Mooley K. P., et al., 2016, ApJ, 818, 105

Mundell C. G., Ferruit P., Nagar N., Wilson A. S., 2009, ApJ, 703, 802

Rees M. J., 1988, Nature, 333, 523 
